\title{
Photosensitive in wide spectral region composites based on polyphenylenevinylene
}

\author{
V. Syromyatnikov ${ }^{1}$, I. Pomaz ${ }^{1}$, A. Verbitsky ${ }^{2}$, Ya. Vertsimakha ${ }^{2}$, S. Nešpůrek ${ }^{3}$, S. Pochekaylov $^{3}$ \\ ${ }^{1}$ Taras Shevchenko Kyiv National University, 64, Volodymyrska str., 01033 Kyiv, Ukraine \\ ${ }^{2}$ Institute of Physics, NAS of Ukraine, 46, prospect Nauky, 03650, Kyiv-39, Ukraine \\ ${ }^{3}$ Institute of Macromolecular Chemistry, Academy of Sciences of the Czech Republic, \\ Heyrovský Sq. 2, 16206 Prague 6, Czech Republic
}

\begin{abstract}
Optical and photovoltaic properties of polyphenylenevinylene derivative poly(2-methoxy-5-(3-,7-dimethyl-octyloxy)-1,4-phenylenevinylene (MDMO-PPV) and its composites with high $(40 \%)$ concentration polymethine dyes (PD) - meso-Cl and hexaindoletricarbocyanine (HITC) films have been studied. Two H-aggregates of dyes with different energies that weakly depend on the molecular structure of dyes, is formed in MDMO-PPV with PD in composites films. The efficiency of photogeneration of charge carrier by these aggregates is greater than the efficiency of photogeneration of charge carriers by the quasi-isolated molecules of dye. It results in substantial expansion of the absorption region (in comparison with the absorption region of molecules in solution) and photosensitivity in the side of higher energies. The presence of PD aggregates in the films MDMO-PPV influences on their photosensitivity that depends on PD molecular structure. This dependence can be caused by interaction between the molecules of MDMO-PPV and dye. In composites MDMO-PPV/HITC, the value of interaction is low and practically does not influence the efficiency of photogeneration of charge carriers by MDMO-PPV molecules, which give a considerable contribution to formation of the photovoltage in $2-3 \mathrm{eV}$ regions. The interaction of meso- $\mathrm{Cl}$ and MDMO-PPV molecules is stronger, and this, probably, results in considerable decrease of photovoltage in the region of the excitation of MDMO-PPV.
\end{abstract}

Keywords: composite, photovoltage, polymer, organic dye, film.

Manuscript received 02.12.08; accepted for publication 18.12.08; published online 30.01.09.

\section{Introduction}

For two last decades, the technology of manufacturing the films of polymeric composites promising for the production of cheap plastic solar cells is being actively developed $[1,2]$.

However, the most effective inorganic solar cells based on $\mathrm{Si}$ and $\mathrm{CuInSe}_{2}$ absorb efficiently the solar irradiation creating charge carriers in the region from 400 up to $1100-1200 \mathrm{~nm}$. At the same time, the best of organic solar devices explored now absorb light with creation of charge carriers only within the range lower 400 to $750 \mathrm{~nm}$ [3]. It is one of the reasons for their smaller efficiency of light transformation by organic solar cells. Therefore, the actual problem is the development of films of the composites photosensitive in a wide spectral range (400-1100 nm) in order to design and produce organic solar devices.
The composites consisting of conductive polymeric compounds and $\mathrm{C}_{60}$ derivatives are studied in the majority of works to make the most effective organic solar cells [3]. In these systems, the effective transfer of a charge occurs between molecules of a polymer and fullerene.

For further enhancement of the efficiency of such solar cells, it is necessary to synthesize new derivatives of $\mathrm{C}_{60}$, which are characterized by a good solubility and a greater diffusion length (lifetime) of photogenerated charge carriers and by a wider region of absorption of solar radiation.

The first of the above-mentioned problems is practically solved, but, to solve the others, it is necessary to search for new materials.

The new strategy aimed at increasing the efficiency of organic solar cells has been proposed in the paper [4]. This strategy consists in the following: at first, to 
develop polymeric composites which will provide the effective light absorption and the photogeneration of charge carriers in the region of 550-1000 nm, where the maximal intensity of the flux of Sun's photons is observed. After that, if necessary, the ways to enhance their photosensitivity and absorbance of sunlight in the short-wave region (400-550 nm) should be developed.

Earlier [4-6] we have developed the physical basis to create the composites from polymethine dyes and carbazol-contained polymers photosensitive in the range $550-950 \mathrm{~nm}$.

The aim of the present paper is the complex study of the composites based on polymethine dyes and conjugated MDMO-PPV polymer as well as experimental checking the possibility to widen the spectral range of photosensitivity of such composites comparing carbazole-containing polymers [4-6].

\section{Experimental}

As a photosensitive component, we used the polymethine dyes which have substituents specially modified to increase their solubility in organic solvents, the maximum of absorption in solutions in the region of $850-1050 \mathrm{~nm}$, and no bulky substituents in the mesoposition [7, 8]. The last circumstance is a necessary requirement, according to [7], for the effective formation of H-type dimer-like aggregates due to the almost flat shape of a molecule.

The most homogeneous and photosensitive films of composites were obtained with the following dyes hexaindoletricarbocyanine (HITC) and meso-Cl (Fig. 1).

HITC and meso-Cl dyes were synthesized in the Institute of Organic Chemistry, NAS of Ukraine [5-7] and MDMO-PPV was produced by Sigma-Aldrich.

The films of composites based on polymethine dyes and MDMO-PPV polymer were prepared by the deposition from their solution in ethylene dichloride by the method of spin casting.
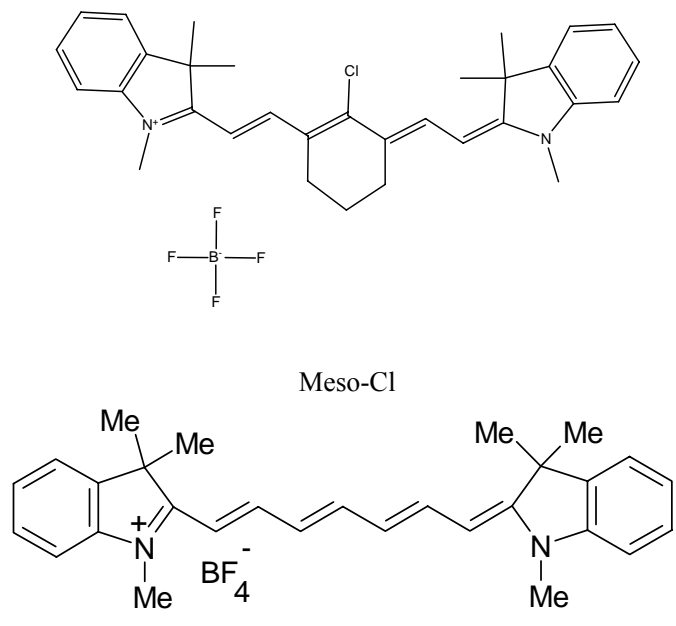

HITC

Fig. 1. Structural formulae of polymethine dyes.
The thickness of studied films was $0.5-1 \mu \mathrm{m}$. For their deposition, two types of substrates were used: glass substrates for the deposition of samples to measure the absorbance and substrates with a conductive ITO layer which was deposited by magnetron sputtering and used as the electrode under measuring the photovoltaic properties of composites.

The absorption spectra were measured with the help of a spectrophotometer "Unicam UV-300" and the spectral dependence of a luminescence and its excitation were measured on a spectrofluorometer "Hitachi".

Photovoltaic properties of composites were measured by the Bergman method improved by Akimov [9]. As an advantage of this method, we indicate that there is no necessity to deposit the top ohmic electrode, for which the preparation technology is not yet designed.

During measurements, a sample was illuminated by modulated monochromatic light $(80 \mathrm{~Hz})$ created with the help of an MDR-4 monochromator. Photovoltage $\left(V_{s}\right)$ was measured by a Unipan 232B nanovoltmeter supplied by a preamplifier with a high input impedance. The spectral dependences of $V$ were normalized on the equal number of incident photons by using a calibrated pyroelement.

\section{Results and discussion}

\subsection{Properties of MDMO-PPV films}

The results of complex studies of spectral dependences of the absorption spectra, spectra of photoluminescence excitation (EPL) and $V_{s}$ of MDMO-PPV films are shown in Fig. 2. It is seen that the absorption spectrum of MDMO-PPV film in the range $2.0-3.4 \mathrm{eV}$ is described by the wide and asymmetrical band with the maximum at $2.61 \mathrm{eV}$ (curve 1).

Nevertheless, two clear maxima at 2.28 and $2.48 \mathrm{eV}$ and shoulder at $2.84 \mathrm{eV}$ are clearly seen on the spectral dependence of $V_{s}$ (curve 2), and maxima at 2.52 and $2.65 \mathrm{eV}$ and a couple of shoulders near 2.3 and $2.9 \mathrm{eV}$ are seen well on EPL spectrum (curve 3 ). $V$ and PL arise only at absorption of light and consequently their values are not influenced with presence of a diffused light.

Hence, presence of extrema on the spectral dependences of $V$ and excitation of PL testifies, that 4 electronic transitions with various efficiency of radiation and photogeneration of charge carriers.

Both diffused light and luminescence give the contribution in a measured signal at the standard technique of the absorption study.

Besides, elementary bands of absorption are strongly overlapped. Therefore, in the absorption one wide band is observed.

To consider electronic processes, in particular processes of light absorption, photogeneration and transport of charge carriers in conductive polymers such as PPV, there are the competition of excitonic and polaronic models (see, for example, [10]).

Occurrence of new bands (electronic states) can be explained by interaction between the neighboring 
polymeric chains (so-called interchain interaction) [11]. This interaction can result in the formation of interchain aggregates [12].

The aggregation in the frameworks of the excitonic model can result in an occurrence of new states caused by the charge transfer in the aggregate - charge transfer states (CT-states) formation [13].

The so-called interchain polarons coupling [14] is considered in the terms of the polaronic model that results in splitting the levels and in occurrence of new bands, for example, in spectra of photoinduced absorption [15].

Since the difference in the energetic position of observable extrema in various spectra does not exceed $3 \%$, it allows us to deconvolute the absorption by Gauss components and to estimate relative efficiency of various transitions. Results of deconvolution are shown in Table 1.

The energies of the extrema, as well as their energies and relative intensities in the different spectra are summarized in this table, too.

The comparison of the intensity of Gaussian components of absorption with the intensity of bands on the spectra of $V$ and EPL (Fig. 2) shows that the efficiency of photogeneration of charge carriers and the efficiency of radiation essentially differ for different

Table 1. Energy position of the maxima on spectral dependences of absorption, PL excitation, $V$ and their ratios for MDMO-PPV and its composites with dyes and in brackets their relative intensity.

\begin{tabular}{|c|c|c|c|c|}
\hline States & 1 & 2 & 3 & 4 \\
\hline $\begin{array}{l}\text { Parameters of } \\
\text { extrema in } \\
\text { the spectra }\end{array}$ & \multicolumn{4}{|c|}{ MDMO-PPV films } \\
\hline D & & & $2.61(1.0)$ & \\
\hline EPL & $\begin{array}{l}2.3 \\
(0.70)\end{array}$ & $\begin{array}{l}2.52 \\
(0.94)\end{array}$ & $2.65(1.0)$ & $2.91(0.45)$ \\
\hline$V_{s}$ & $\begin{array}{l}2.28 \\
(0.99) \\
\end{array}$ & $2.44(1.0)$ & $\begin{array}{l}2.58 \\
(0.71) \\
\end{array}$ & $2.79(0.71)$ \\
\hline$V_{s} / D$ & $\begin{array}{l}2.26 \\
(1.0)\end{array}$ & - & - & $2.86(0.35)$ \\
\hline $\mathrm{EPL} / \mathrm{D}$ & $\begin{array}{l}2.26 \\
(1.0)\end{array}$ & $\begin{array}{l}2.52 \\
(0.85)\end{array}$ & $\begin{array}{l}2.65 \\
(0.88)\end{array}$ & - \\
\hline $\begin{array}{l}\text { Gaussian } \\
\text { components } \\
\text { of } D\end{array}$ & $\begin{array}{l}2.30 \\
(0.43)\end{array}$ & $\begin{array}{l}2.48 \\
(0.98)\end{array}$ & $\begin{array}{l}2.65 \\
(0.81)\end{array}$ & $2.85(1.00)$ \\
\hline \multicolumn{5}{|c|}{$\begin{array}{c}\text { HITC+MDMO-PPV composite films } \\
\end{array}$} \\
\hline $\bar{D}$ & & $\begin{array}{l}2.58 \\
(0.99)\end{array}$ & & $2.8(1.0)$ \\
\hline $\bar{V}$ & $\begin{array}{l}2.27 \\
(0.99)\end{array}$ & $2.46(1.0)$ & & $2.84(0.63)$ \\
\hline$V / D$ & $\begin{array}{l}2.24 \\
(1.0) \\
\end{array}$ & $\begin{array}{l}2.45 \\
(0.62) \\
\end{array}$ & & $2.84(0.42)$ \\
\hline \multicolumn{5}{|c|}{ Meso-CI+MDMO-PPV composite films } \\
\hline$D$ & & $\begin{array}{l}2.52 \\
(0.97)\end{array}$ & & $2.78(1.0)$ \\
\hline$V$ & & & $\begin{array}{l}2.66 \\
(0.48)\end{array}$ & $2.8(1.0)$ \\
\hline$V_{s} / D$ & & & $\begin{array}{l}2.66 \\
(0.45)\end{array}$ & $2.90(1.0)$ \\
\hline
\end{tabular}

states. So, the highest efficiency of photogeneration of charge carriers is observed for the lowest state with the energy $2.26 \mathrm{eV}$, and is minimal for the state with the energy $3.2 \mathrm{eV}$. On the contrary, the efficiency of radiation is maximal for the states with energies 2.48 and $2.65 \mathrm{eV}$. For convenience of the analysis, in Table 1 we summarized energies of maxima in various spectra and their intensities concerning a maximum in each spectrum (in brackets).

PL with a maximum near $2.11 \mathrm{eV}$ and smooth increase in the region of low energy on which the shoulders at about 2.0 and $1.85 \mathrm{eV}$ are well-expressed is observed in spectra of a photoluminescence MDMOPPV films and composites.

Introduction of dye practically does not influence energy position of PL maxima and shoulders, but results in the decrease of PL intensity.

Intensity of $\mathrm{PL}$ in its peak decreases at introduction of HITC by 1.7 times, and meso-Cl - almost by 4 times.

It is possible to explain this essential decrease only partially by absorption of radiation of molecules MDMO-PPV by the aggregates of dyes since in this region their absorption are low. Hence, it is necessary to assume that it is also caused by weak interaction of molecules MDMO-PPV and dye, which is stronger for molecules MDMO-PPV and meso-Cl.

The deconvolution on the Gaussian components for PL spectra shown, that PL band is well described by the

$$
\text { V, EPL a.u. D, a.u. }
$$

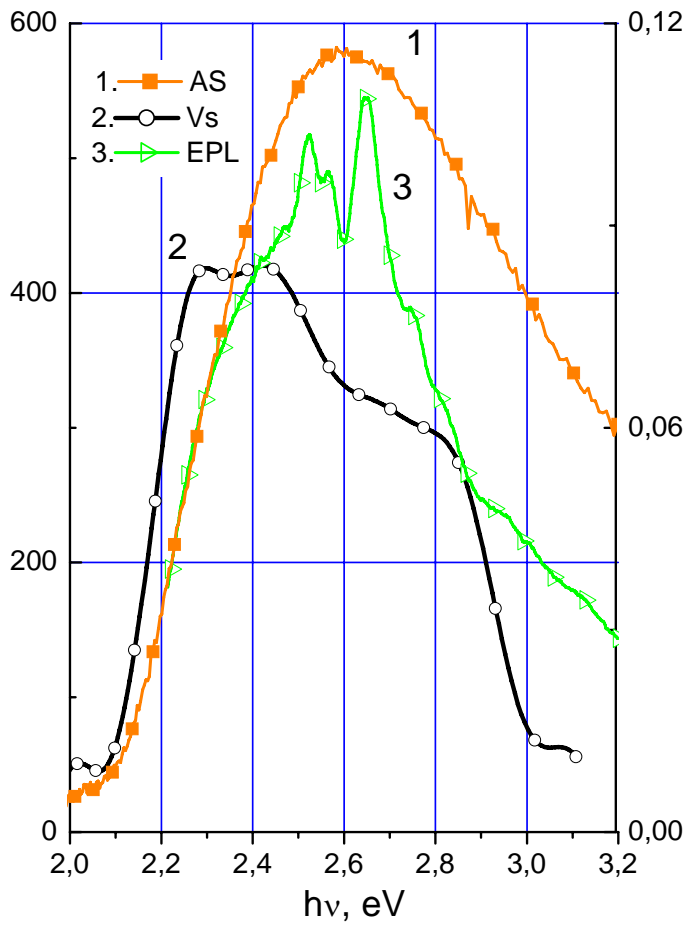

Fig. 2. Spectral dependences of absorption $D(1), V_{s}(2)$ and PL excitation EPL (3) measured at the equal energy of incident monochromatic illumination. 
electronic transition at $2.11 \mathrm{eV}$ and intramolecular oscillation with the energy $0.13 \pm 0.01 \mathrm{eV}$. Since PL is caused by transitions from the lowest excited state to the highest occupied state, it is possible to assume that the density of states in the valence band of MDMO-PPV will consist of three narrow levels, two of which with lower density are related with intramolecular oscillations. This is typical for organic crystals with a weak interaction between molecules.

\subsection{Influence interaction of molecules of dye and polymer on properties MDMO-PPV films}

From mentioned in the previous section it is clear that the introduction of molecules of dye or their aggregates which absorb light in the region of a transparency MDMO-PPV films can result both in partial absorption of a diffused light and a luminescence, and an occurrence of the interaction between molecules of MDMO-PPV and dye. Partial absorption of light by dye aggregates should lead to the occurrence of structure in spectra of absorption of composites and practically not influence spectral dependence of $V$ in the region of absorption MDMO-PPV films since it is defined only by light absorbed by MDMO-PPV molecules. On the other hand weak interaction between molecules of MDMOPPV and dye should not influence practically spectra of absorption of MDMO-PPV films, but can influence essentially properties of electronic states of MDMOPPV, for example, on efficiency of generation by them of charge carriers and radiation.

The results of complex measurement of MDMOPPV films properties with the high concentration of two various dyes are resulted in Figs 3 and 4.

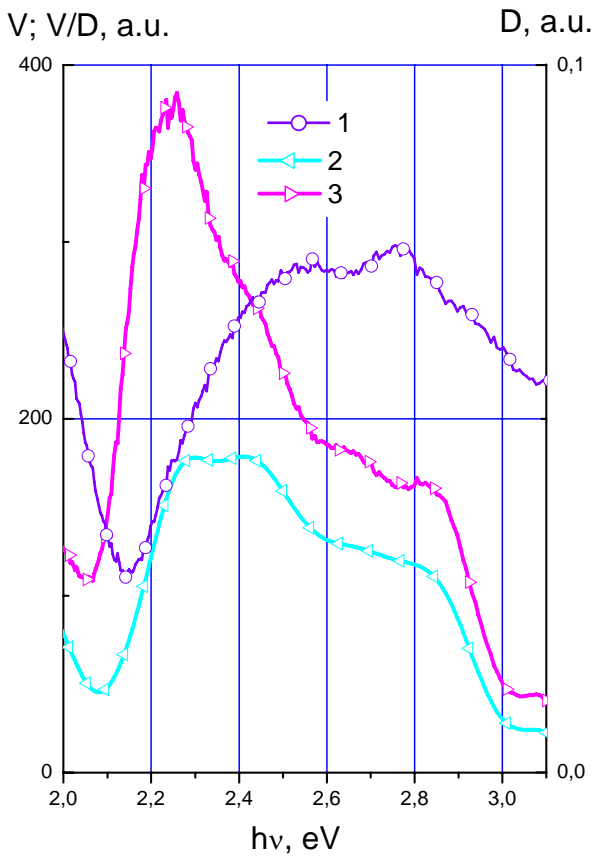

Fig. 3. Spectral dependences of optical density $D(1)$, photovoltage $V(2)$ and their ratios $V / D(3)$ for films of composites MDMO-PPV+HITC.

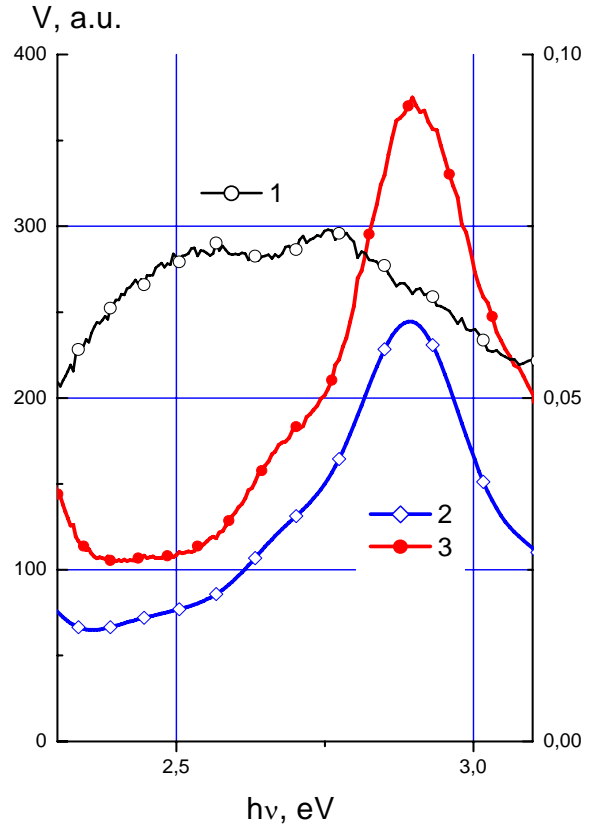

Fig. 4. Spectral dependences of optical density $D(1)$, photovoltage $V(2)$ and their ratios $V / D(3)$ for films of composites MDMO-PPV+meso-Cl.

From Figs 3 and 4 it is obvious that in spectra of absorption composite films two weakly expressed maxima at about 2.54 and $2.8 \mathrm{eV}$ instead of a maximum $2.61 \mathrm{eV}$ in MDMO-PPV films practically irrespective of molecular structure of dye, i.e. the deposit of the influence of absorption by luminescent and a diffused light is observed in the both composites. At the same time, the comparison of Figs 2 and 3 shows that the spectra of $V_{s}$ are similar for films of HITC+PPV and PPV films are similar, i.e. the properties of the barriers between aggregates HITC and PPV and consequently the efficiency of charge carriers by PPV molecules practically doesn't changed.

Although the comparison of Figs 2 and 4 shows that the efficiency of charge carriers photogeneration by PPV states 2.26 and $2.54 \mathrm{eV}$ sufficiently decreased in composites PPV-meso-Cl. Due to this only maximum at $2.28 \mathrm{eV}$ and shoulder at $2.65 \mathrm{eV}$ are observed in $V_{s}$ spectra of PPV-meso-Cl composites, i.e. the interaction of the molecules PPV and meso-Cl didn't influence only the state at $2.8 \mathrm{eV}$. This is correlated with the observed significant decrease of PL for PPV-meso-Cl composites. Thus, the interaction of the molecules PPV and meso-Cl mainly leads to the decrease of charge carrier photogeneration by low energetic states of PPV molecules 2.26 and $2.54 \mathrm{eV}$.

\subsection{Properties of composites}

The spectrum of absorption of dyes HITC and meso-Cl in a solution consists of a narrow intensive bands with a maxima at 1.64 and $1.58 \mathrm{eV}$ with width 0.1 and $0.08 \mathrm{eV}$, respectively, and weak absorption band at about 1.8 and $1.75 \mathrm{eV}$, respectively, caused by intramolecular oscillations (Figs 5 and 6). 
Naturally, owing to interaction with molecules MDMO-PPV in a film of a composite the maximum of their absorption is displaced in the region of smaller energy on 0.05 and $0.075 \mathrm{eV}$, respectively. It testifies that meso-Cl molecules interact with molecules MDMOPPV more strongly than molecules HITC. This is correlated well with marked in section 3.2 stronger influence of meso-Cl molecules with electronic states of MDMO-PPV with energies 2.26 and $2.54 \mathrm{eV}$. Additional absorption, the intensity of which is much higher than that of weak absorption in a solution, is caused by intramolecular interaction (Figs 5 and 6) and is simultaneously observed in spectra of absorption of composite films in the range of $1.7-2.15 \mathrm{eV}$.

Three peaks are observed in spectra $V_{s}$ of composites HITC+MDMO-PPV at energies 1.62, 1.77 and $1.95 \mathrm{eV}$ which, certainly, are more precisely visible in spectra of $V_{s} / D$ (Fig. 6, curve 4). Energy position of peak $1.62 \mathrm{eV}$ practically coincides with the energy of a maximum of absorption for HITC solution, i.e. energy of a quasi-isolated molecule of this dye, and can be caused by photogeneration of charge carriers by these HITC molecules. Peaks of $V_{s}$ in the region of additional absorption at 1.77 and $1.95 \mathrm{eV}$ can be caused by formation of two types of aggregates. Propensity of this type of dyes to formation of $\mathrm{H}$-aggregate type was revealed in high-concentrated solutions [8], and later we confirmed this fact experimentally for composites of this type of dyes with carbazole polymers $[6,16]$. As it is visible from the spectral dependence $V_{s} / D$ in Fig. 6 (curve 4), the efficiency of photogeneration of charge carriers for these aggregates is higher than that of photogeneration of charge carriers from quasi-isolated HITC molecules, as results in expansion of spectral range of $V_{s}$ in the short-wavelength region.

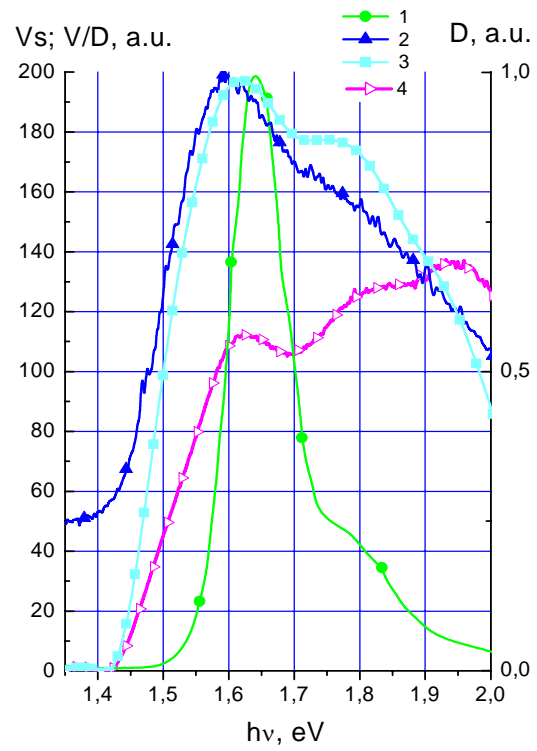

Fig. 5. Spectral dependences of optical density $D$ of solution in dichloroethane HITC (1), a film (2), photovoltage $V(3)$ and their ratio $V / D$ (4) for films of composites MDMO-PPV+HITC.

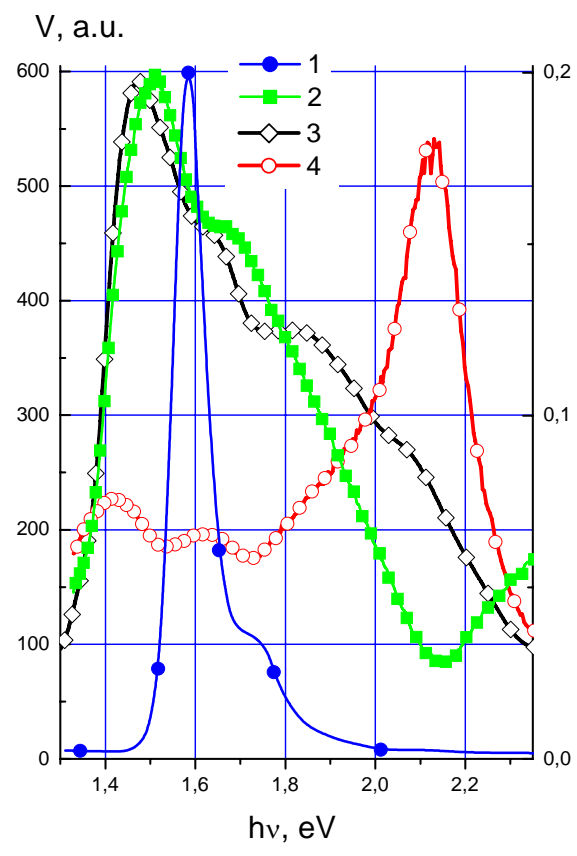

Fig. 6. Spectral dependences of optical density $D$ of a solution dichloroethane (1), a film (2), photovoltage $V$ (3) and their ratio $V / D(4)$ for films of composites MDMO-PPV+meso-Cl.

Already precisely 4 peaks with maxima near 1.47 , 1.65, 1.88 and $2.13 \mathrm{eV}$ are seen in spectra $V_{s}$ of composites meso-Cl+MDMO-PPV. Thereof, the peak $1.65 \mathrm{eV}$ corresponds to photogeneration of charge carriers by quasi-isolated meso- $\mathrm{Cl}$ molecules, and 1.88 and $2.13 \mathrm{eV}$ - to the formation of two types of dye aggregates (most probably of H-type).

For convenience of parameters comparison of both dyes composites in Table 2 energy positions of extrema in their spectra of absorption, $V_{s}$ and $V_{s} / D$ and intensity concerning the peak caused by presence quasi-isolated molecules of dye in composites are resulted.

From the table, it is visible that the first feature of meso-Cl+MDMO-MDMO-PPV composite is more high efficiency of photogeneration of charge carriers of $2.13 \mathrm{eV}$ state (Fig. 6, curve 4) in comparison with efficiency of their photogeneration of quasi-isolated molecules meso-Cl and aggregates in composites HITC+MDMO-PPV. The second feature of composites meso-Cl+MDMO-PPV is presence in spectra $V_{s}$ and $V_{s} / D$ peak $1.45 \pm 0.02 \mathrm{eV}$, i.e. at energies that are less than the energy of isolated molecules meso-Cl. Therefore, it is natural to connect the formation of this peak with the formation of charge transfer complex (CTcomplex) between molecules meso-Cl and MDMO-PPV. Formation of similar CT-complexes were earlier observed in composites of carbazole-contained polymers with the same polymethine dyes [4] and polyepoxypropylcarbazole with TNF [13]. CT-complexes displaying in spectra of $V_{s}$ and $V_{s} / D$ in composites meso$\mathrm{Cl}+\mathrm{MDMO}-\mathrm{PPV}$ is quite logical, since the energy of interaction of molecules meso- $\mathrm{Cl}$ and MDMO-PPV is higher than molecules HITC and MDMO-PPV. 
Table 2. Energy position of extrema on spectral dependences of absorption, photovoltage and their ratios for films of composites of dyes and in brackets of their intensity with respect to the state of a qusi-isolated molecule.

\begin{tabular}{|c|c|c|c|c|c|c|c|}
\hline $\begin{array}{c}\text { Compo- } \\
\text { site }\end{array}$ & \multicolumn{2}{|l|}{ HITC+MDMO-PPV } & \multicolumn{5}{l|}{ Meso-Cl+MDMO-PPV } \\
\hline Extremum & 1 & 2 & 3 & - & 1 & 2 & 3 \\
\hline$D$ & 1.58 & & & & 1.51 & & \\
& $(1.0)$ & & & & $(1.0)$ & & \\
\hline$V_{s}$ & 1.61 & 1.77 & 1.95 & 1.47 & 1.65 & 1.87 & 2.10 \\
& $(1.0)$ & $(0.9)$ & $(0.62)$ & $(1.0)$ & $(0.77)$ & $(0.62)$ & $(0.39)$ \\
\hline$V_{s} / D$ & 1.62 & 1.77 & 1.95 & 1.42 & 1.61 & 1.88 & 2.13 \\
& $(1.0)$ & $(1.09)$ & $(1.22)$ & $(1.17)$ & $(1.0)$ & $(1.19)$ & $(2.78)$ \\
\hline
\end{tabular}

Comparison of photovoltage spectra of studied in this work composites HITC+MDMO-PPV with composites HITC developed by us earlier [15] with carbazole polymers (Fig. 7) and composites based on MDMO-PPV with $\mathrm{C}_{60}$ derivatives shows that researched composites HITC+MDMO-PPV allow further expansion of the photosensivity spectral range and can perspective material for development of plastic solar cells. Observable weak photosensivity of composites HITC+MDMO-PPV can be increased by optimization of manufacturing techniques for these films and/or by the replacement of HITC with other polymethine dye with the higher efficiency for the formation of H-aggregates in MDMO-PPV.

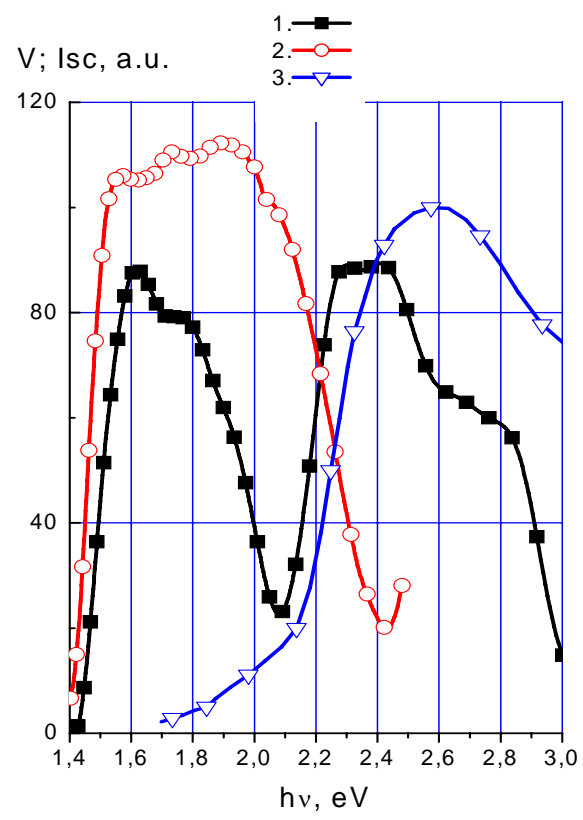

Fig. 7. Spectral dependences $V_{S}$ of composites MDMOPPV+HITC (1), I-VC-OMA+HITC [16] (2), and MDMO$\mathrm{PPV}+\mathrm{PCBM}[3,4](3)$.

\section{Conclusions}

In the region of dye weak absorption $(1.4-2.1 \mathrm{eV})$ in their composites with MDMO-PPV, formed are on two types of $\mathrm{H}$-aggregates with various energies, in which the efficiency for photogeneration of charge carriers is higher than that for photogeneration of charge carriers from their quasi-isolated molecules. It results in essential expansion of the absorption spectral region (in comparison with the range of absorption of molecules in a solution) and photosensivity short wavelength side from 1.6 up to $2 \mathrm{eV}$. Besides, in composites meso-Cl which molecules interact with molecules MDMO-PPV more effectively, the photosensitive complex with charge transfer possessing the energy $1.45 \pm 0.02 \mathrm{eV}$ is formed. The latter energy is less than the minimal energy of excitation for a dye molecule.

Besides, the introduction of dyes in the film of MDMO-PPV composites results in appreciable changes of efficiency of photogeneration of nonequilibrium charge carriers of separate states of MDMO-PPV, which depends on molecular structure of dyes. In composites MDMO-PPV/HITC interaction between their molecules small, also the essential contribution to occurrence of a photovoltage in short wavelength region $(2-3 \mathrm{eV})$ is observed. The interaction of MDMO-PPV with molecules meso- $\mathrm{Cl}$ stronger, that results in strong reduction of efficiency of photogeneration of charge carriers of at excitation of MDMO-PPV states at 2.3 and $2.48 \mathrm{eV}$.

The spectral region of photosensitivity of composites HITC/MDMO-PPV is wider than carbazolebased polymer composites with HITC and PPV with $\mathrm{C}_{60}$ derivatives. Therefore composites HITC/MDMO-PPV can be used for development of plastic photoconverters, including the solar cells sensitive in wide spectral range (from 400 up to $900 \mathrm{~nm}$ ).

Work is executed according to the Ukrainian-Czech project in 2008.

\section{References}

1. C.J. Brabec, N. Sariciftci, Solarzellen aus Plastik // Erneuerbare Energien 2, p. 28-34 (1997).

2. I.A. Akimov, A.M. Meshkov, I.Yu. Denisyuk, Nanostructured composite organic semiconductors // Functional Materials 5, p. 363-369 (1998).

3. C.J. Brabec, S.N. Sariciftci, J.C. Hummelen, Plastic solar cells // Adv. Funct. Mater. 11, p.15-26 (2001).

4. J.G. Grote, Ya.I. Vertsimakha, The photosensitive polymeric composites from inorganic and organic semiconductors and prospects of their use // Proc. of the XIV Intern. Symposium "Advance Display Technologies". October 10-14, 2005, Crimea, Ukraine, p. 216-224.

5. S. Studzinsky, V. Syromyatnikov, A. Ishchenko, N. Derevyanko, Ya. Vertsimakha, A. Verbitsky, Effect of polymer matrix on photosensitivity of polymethine dye based composites // Nonlinear Optics, Quantum Optics 33(1-2), p.151-159 (2005). 
6. N. Beresina, V. Syromyatnikov, A. Ishchenko, A. Verbitsky, Ya. Vertsimakha, Effect of polymer matrix on photosensitivity of meso- $\mathrm{Cl}$ polymethine dye based composites // Functional Materials 13 (4), p. 676-680 (2006).

7. A.A. Ishchenko, Structure, Spectral and Luminescent Properties of Polymethine Dyes. Naukova dumka, Kiev, 1994 (in Russian).

8. A.A. Ishchenko, Physicochemical aspects of the creation of modern light-sensitive materials based on polymethine dyes // Theoretical and Experimental Chemistry 34 (4), p. 191-210 (1998) (in Russian).

9. I.A. Akimov, The study of internal photoeffect in semiconductors by capacitor method // Optikomekhanicheskaya promyshlennost' No. 5, p. 4-13 (1966) (in Russian).

10. A.J. Heeger, Nature of the primary photoexcitations in poly(arylene-vinylenes): Bound neutral excitons or charged polaron pairs, in Primary excitation of conjugated polymers: Molecular exciton versus semiconductor band model (Ed. N.S. Sariciftci). World Scientific, Singapore, 1997.
11. Thuc-Quyen Nguyen, Vinh Doan, and B.J. Schwartz, Conjugated polymer aggregates in solution: control of interchain interactions // J. Chem. Phys. 110, p. $4068-4078$ (1999).

12. M. Aguiar, M.C. Fugihara, I.A. Hümmelgen, L.O. Peres, J.R. Garcia, J. Gruber, L. Akcelrud, Interchain luminescence in poly(acetoxy-p-phenylene vinylene // J. Luminescence 96, p. 219-225 (2002).

13. M. Pope and C.E. Swenberg, Electronic Processes in Organic Crystals. Clarendon, Oxford, 1982.

14. A. Cravino, H. Neugebauer, S. Luzzati, M. Castellani, A. Petr, L. Dunsch and N.S. Sariciftci // J. Phys. Chem. B 106, p. 3583-3592 (2002).

15. C. Winder and N.S. Sariciftci, Low bandgap polymers for photon harvesting in bulk heterojunction solar cells // J. Mater. Chem. 14, p. 1077-1086 (2004).

16. A. Ishchenko, N. Derevyanko, Yu.P. Piryatinskii, A. Verbitsky, D. Filonenko, S. Studzinsky, Optical and photovoltaic properties of films and polymer composites based on near infra-red polymethine dye // Materials Science 20 (4), p. 5-12 (2002). 